\title{
Both the microbiome and the macrobiome can influence immune responsiveness in psoriasis
}

\author{
MAGDALENA KIERASIŃSKA, KATARZYNA DONSKOW-EYSONIEWSKA
}

Laboratory of Parasitology, General Karol Kaczkowski Military Institute of Hygiene and Epidemiology, Warsaw, Poland

\begin{abstract}
It is debatable whether intestinal dysbiosis in autoimmune disease is a cause or a consequence of chronic inflammation, but it is known that intestinal dysbiosis in the course of the disease is accompanied by an increased number of pro-inflammatory lymphocytes in the Th17 population. Yet, little is known about the systemic implications of skin and even the intestinal microbiome for skin immunity and pathogenesis in psoriasis, which the most prevalent autoimmune disease in the Caucasian population. The pathogenesis of psoriasis is multifactorial with notable contributions from genetics and environmental factors (e.g. diet, drugs and infection). This article describes alterations in the microbiome and macrobiome, which are involved in immune regulation. The composition of the gut microbiome can dramatically affect immune development and affect susceptibility to diseases, especially autoimmune disorders such as psoriasis. Understanding the mechanisms of pathogenesis induced by the micro- and macrobiome may prove crucial for innovative future solutions in skin disease treatment.
\end{abstract}

Key words: psoriasis, microbiota, T cells, parasites.

(Cent Eur J Immunol 2021; 46 (4): 502-508)

\section{Introduction}

The human genome can indicate predisposition to certain diseases, but there are many environmental factors that can promote or even inhibit development, including epigenetic factors. One of the most important factors influencing the development of organisms is bacteria.

These microorganisms are everywhere, in food, in water and they can colonize the body, forming microbiota on the skin or in the digestive system. During long-term co-evolution, mutualism has developed between a host and its microbiota. Up to 1000 species colonize the gut of a healthy human adult, together with a variety of fungi, viruses, and archaea [1]. There is a mutual dependence where bacteria depend on the host environment and its nutrients [2]. In return, the human microbiome not only provides the host with the necessary metabolites, but also regulates its immune system, and maintains overall human health. The occurrence of these microorganisms in the human body creates homeostasis between the host and its microbiota $[1,3]$.

Understanding the relationship between the body and its microbiome can be key to understanding the mechanisms of autoimmunity. Some viral, bacterial, or parasitic infections in people with specific genetic backgrounds and immune disorders may trigger autoimmunity and lead to the development of autoimmune diseases [4]. The microbiome influences various inflammatory and systemic diseases such as type 1 diabetes mellitus, rheumatoid arthritis, inflammatory bowel disease, and even psoriasis ( $\mathrm{PsO})$ [3].

The pathogenesis of $\mathrm{PsO}$ is multifactorial, with genetics and environmental factors such as lifestyle, diet, and infection [5-7] playing a significant role. According to the National Psoriasis Foundation (NPF), PsO is the most prevalent autoimmune disease present in cold areas of the world, affecting about 1-3\% of the Caucasian population. $\mathrm{PsO}$ is a chronic inflammatory skin disease with a particularly negative impact on a patient's mental health. PsO ranges in severity from the presence of a few scattered, sharply demarcated, scaly, red, coin-sized skin lesions or scaly plaques, to involvement of almost the entire body surface. Plaque psoriasis (psoriasis vulgaris), commonly referred to as $\mathrm{PsO}$, is the most common form, although there are four other phenotypic conditions: inverse (PsO of intertriginous areas such as the armpits, groin, or other skin folds), erythrodermic (a generalized form of inflammatory $\mathrm{PsO}$ that affects most body sites), pustular (PsO that is characterized by deep pustules on palms and soles), and guttate (distinctive $\mathrm{PsO}$ plaques of 0.5 to $1.5 \mathrm{~cm}$ in diameter, often seen in children and young adults) [8]. Although not life-threatening, it causes tremendous morbidity

Correspondence: Magdalena Kierasińska, Laboratory of Parasitology, General Karol Kaczkowski Military Institute of Hygiene and Epidemiology, Warsaw, Poland, e-mail: magdalena.glaczynska@wihe.pl

Submitted: 17.03.2021; Accepted: 02.08.2021 
and personal angst for patients. Stigmatization constantly accompanies patients and influences their family, social and occupational lives. The abnormal lifestyle of patients, resulting, inter alia, in social stigma, has a significant effect on depression and cardiovascular risk [9].

The main clinical picture is the thickened skin covered in scales caused by the rapid proliferation of skin cells. Unfortunately, the pathogenesis of PsO is still not fully understood, although it seems that T-helper 17 (Th17) cells and the cytokines they produce, such as interleukin (IL)-17, IL-22 and IL-23, may play a critical role in the pathogenesis of $\mathrm{PsO}[7,10]$.

\section{Immune response in psoriasis}

Although immune cells are relatively sparse in skin, recent reports have led to a reclassification of $\mathrm{PsO}$ from a skin disease to a T-cell mediated disease. The reason for the paradigm change is an observed increase in expansion and stimulation of the population of Th1, Th17 and T-helper 22 (Th22) lymphocytes, producing inflammatory cytokines, including tumor necrosis factor $\alpha$ (TNF- $\alpha$ ), IL-17 and IL-22 [7, 11].

It seems that the most important factor in developing autoimmune diseases is cross-talk between the innate and adaptive immune systems. The IL-23/IL-17 axis, TNF- $\alpha$, dendritic cells (DCs) and keratinocytes $[9,12]$ all contribute; DCs, along with macrophages, B and T lymphocytes, secrete IL-23, which stimulates Th17 cells to differentiate and proliferate [13]. Furthermore, strong activation of Th17 cells stimulated by IL-23 leads to skin inflammation and hyperproliferation of keratinocytes [7, 12-14].

In response to a stressor factor, such as physical trauma or antigen stimuli, DCs in the skin are activated and present the antigen to nadve T lymphocytes [9, 13]; as a result, activated $\mathrm{T}$ lymphocytes differentiate into type Th1, type Th17, and type Th22 cells [9]. Disturbed levels of inflammatory molecules such as C-reactive protein, fibrinogen, interferon $\alpha$ (IFN- $\alpha$ ), interferon $\gamma$ (IFN- $\gamma$ ), IL-2, IL-6, IL-8, IL-12, IL-15, and IL-18 may be responsible for the development of comorbidities $[9,15,16]$. This dysregulation in patients with $\mathrm{PsO}$ can promote the development of atherosclerosis, and contribute to the development of insulin resistance and type II diabetes mellitus [9]. Additionally, elevated levels of cytokines may influence the development of depression (with a prominent inflammatory role of IL-6) [9, 17].

Th17 cells have been found to play a significant role in the pathogenesis of autoimmune diseases including PsO. Th17 cells produce many cytokines such as IL-17, IL-22, IL-21 and TNF- $\alpha$. Interleukin 17 is involved in many inflammatory processes including the recruitment of neutrophils and induces the expression of proinflammatory cytokines such as IL-6, IL-8 and CXCL8. Elevated levels of these factors are observed in PsO [18]. Further, IL-17 induces Th22 cells and the production of antimicrobial peptides in keratinocytes. Activated Th22 cells produce IL-22, which can increase keratinocyte hyperplasia [7].

\section{Increased reactivity of keratinocytes}

Human skin has an area of around $1.8 \mathrm{~m}^{2}$ and is the major barrier and provides one of our first lines of defense against the external environment. Both keratinocytes and immune cells play an important role in responding to danger signals. They participate in the mechanisms of the innate and adaptive immune responses (by production of innate effector molecules and by activation of $\mathrm{T}$ cells by producing cytokines) [7]. Keratinocytes produce IL-20 and antimicrobial peptides such as cathelicidin LL-37, defensins and S100 protein [19]. Therefore, exacerbation of PsO symptoms may be caused by external factors such as infection or damage [7]. Interleukin 20 is the growth factor for keratinocytes and induces them to produce TNF- $\alpha$ and IL- 6 and the chemokine ligand 20 (CCL20) [18]. CCL20 is a strong chemokine for lymphocytes and DCs, but has a lower effect on neutrophils. Recruited inflammatory DCs and T cells in skin lesions start to produce IL-17, IFN- $\gamma$ and IL-22 [7]. As a result, keratinocytes are activated and are characterized by increased resistance to apoptosis. The impact of bacterial infections shows that microbial factors such as lipopolysaccharides (LPS), or TNF- $\alpha$ and IFN- $\gamma$ can induce expression of CCL20. Interleukin 10 inhibits CCL20. Additionally, the infection causes an increase in the production of TNF- $\alpha$ and IFN- $\alpha$. TNF- $\alpha$ in turn activates neutrophils by stimulating keratinocytes to produce the CXCL8 chemokine [7, 18].

\section{Increased reactivity of dendritic cells}

Dendritic cells play one of the most important roles in the course of $\mathrm{PsO}$; they are able to provoke inflammatory responses and induce immune tolerance. Two types of cells can be observed in the skin: epidermal DC (myeloid DCs) and plasmacytoid DCs (pDCs), both populations can stimulate T cells to produce IL-17 and IFN- $\gamma$ [7]. They are able to distinguish between microbiota and pathogens because they are equipped with a wide range of pattern recognition receptors (PRRs), including toll-like receptors (TLRs) and Nod-like receptors (NLRs) [20]. During inflammation, DCs in the intestine and the draining mesenteric lymph nodes provide proinflammatory cytokines, such as IL-6 and IL-23, which are suggested to contribute toward Th17 cell maturation [21]. Also, in PsO, the DC subsets are modified compared to healthy skin: Langerhans cells (LCs) are markedly reduced, whereas pDCs are increased in lesions. pDCs in healthy skin are almost absent, but they are abundantly present in the skin of psoriatic plaques, particularly in early lesions. There they become activated and produce IFN- $\alpha$, which is one of the inflammatory mediators essential for the development of PsO lesions [20]. 


\section{Intestinal microbiota in psoriasis - up or down?}

Organisms have developed special mechanisms to prevent microorganisms from entering the circulation, where they could induce a systemic inflammatory response [22]. The first obstacle is the physical gastrointestinal barrier that prevents bacteria from penetrating into deeper tissues. It is made of a protective layer of mucus and tight junctions.

Another safety barrier is the immunological defense barrier. It consists of specific and nonspecific elements (e.g. sIgA, antimicrobial peptides) of the immune system that form gut-associated lymphoid tissue (GALT) [23, 24] and skin [25]. The microbiome is a natural component of the defense mechanisms. It is debatable whether microbiota dysbiosis in autoimmune disease is a cause or a consequence of chronic inflammation, but it is known that intestinal dysbiosis in the course of the disease is accompanied by an increased number of pro-inflammatory lymphocytes in the Th17 population.

Segmented filamentous bacteria (SFB) were reported to induce Th17 cells in autoimmune responses [26].

It has been observed that bacteria of the genera Lactobacillus and Bifidobacteria produce numerous bactericides, such as lactic acid, bacteriocins, bacteriocin-like substances (BLIS) and short-chain fatty acids (SCFA) [24]. In addition, metabolites of gut microbes such as SCFA are involved in communication between the gut microbiota and the immune system. SCFA reduce the development of intestinal inflammation by inhibition of histone deacetylase (HDAC), which leads to increased acetylation of histone $\mathrm{H} 3$ in the promoter region of the Foxp3 gene, which is characteristic of Treg lymphocytes, and activation of Treg [27].

The gut microbiota plays a crucial role in human health and disease with a key influence on the development and maintenance of immune homeostasis of the host [28]. The gut microbiota is shaped by several environmental factors, including diet, infectious agents, parasitic infection and antibiotic treatment. The composition of the microbiome varies among individuals, but it is possible to identify some sources of variation $[1,3]$.

The intestinal microbiota belong to nine types, the most numerous of which are Firmicutes and Bacteroidetes [29]. Apart from bacteria, the composition of microbiota also includes yeasts, viruses and archaea [23]. As a result of dysbiosis, there is abnormal activation of PRRs and the production of cytokines (IFN- $\gamma$, IL-18, IL-22) [1]. This condition is associated with many diseases, especially those related to the digestive system and the immune system [29]. Microbiome perturbations have been associated with autoimmune-mediated diseases such as asthma, atopic dermatitis, and multiple sclerosis [30]. In addition, the immunoregulatory effect of the physiological intestinal microbiota on the host cells is realized primarily through the structural components of bacteria cells and with the use of their metabolites [27, 31]. The immune system is educated through inflammatory factors, such as LPS and lipoteichoic acid (LTA), secreted by Gram-negative and Gram-positive bacteria, respectively. It causes, inter alia, the stimulation of peripheral regulatory $\mathrm{T}$ lymphocytes partly dependent on the T-cell receptor (TCR) [9].

The human gut microbiota and the skin microbiota are among the most studied to date $[9,32,33]$. Some research indicates that intestinal microbiota dysbiosis may play a key role in the development of psoriatic disease due to an aberrant inflammatory response that can be connected with the skin [9]. Relative changes in the gut microbiome of patients with PsO of the skin as well as patients with psoriatic arthritis (PA), compared to healthy people, have been reported $[9,33]$.

Patients with $\mathrm{PsO}$ and $\mathrm{PA}$ have a significant decrease in microbial gut diversity [28, 33]. Patients with PsO have microbiota characterized by a reduction in Bacteroides and Proteobacteria, and increased proportions of Actinobacteria and Firmicutes [25, 31, 32], whereas PA patients showed a reduced number of bacteria from Akkermansia spp., Ruminococcus spp. and Pseudobutyrivibrio spp. compared with healthy controls [34]. Interestingly, other research indicates the opposite results. According to Codoner et al. the psoriatic microbiome was characterized by an increased presence of Faecalibacterium spp. and a decrease of Bacteroides spp. [35] Bacteroides are known to play an immunomodulatory role in the gut through the production of polysaccharide A, which activates regulatory T cells [9]. Additionally, the prevalence of Actinobacteria was negatively correlated with $\mathrm{PsO}$ area and severity index (PASI). Also, the increase in the Firmicutes to Bacteroidetes ratio in PsO subjects was positively correlated with PASI [28, 36]. Several studies have shown that high proportions of Firmicutes, like Bacteroidetes, affect carbohydrate metabolism. These bacteria increase the production of acetates, at the same time reducing the production of butyrate, medium and short chain fatty acids (SCFA). Reducing the production of SCFA can cause inflammation and weaken the intestinal epithelial barrier. The integrity of the gut epithelial barrier directly affects the process of antigen presentation and is responsible for maintaining the immune balance. The weakening of the gut epithelial barrier affects both local and systemic immune responses [27]. The conflicting results could possibly be due to differences in the applied research methods and/or patient demographics.

In cohort studies of patients with PsO, the genera Actinomyces and Sutterella dominated the intestines. Sutterella is proinflammatory and has been associated with the occurrence of inflammatory bowel disease. Therefore, it is very possible that, along with other bacteria, they may play a role in the development of PsO symptoms [34]. These observations may explain how bacteria disrupt the 'gutblood' barrier and affect the immune system. 
The genus Faecalibacterium represent about $5 \%$ of the gut bacterial population and remarkably its increase in the gut microbiota has been associated with immune regulation [37]. One of its properties is the ability to produce butyrate, which has anti-inflammatory properties through inhibiting the NF- $\kappa \mathrm{B}$ pathway [9]. There is a negative correlation between the abundance of Faecalibacterium $(F$. prausnitzii) in inflammation and irritable bowel syndrome and celiac disease, among others. Other studies have found that an increase in species belonging to this genus is associated with inflammatory diseases such as Crohn's disease [38]. Dysbiosis in Faecalibacterium subspecies has been shown to impact the gut epithelial barrier and, as a result, influence the development of atopic dermatitis [35, 39]. Some studies show that Faecalibacterium was significantly lower in patients with $\mathrm{PsO}$ while Akkermansia was constant [28]. Furthermore, some studies show that a similar dysbiosis occurs in the gut microbiome in both IBD (Crohn's disease and ulcerative colitis) and PsO patients $[9,40]$. In patients with PsO, PA and Crohn's disease, there is a significantly reduced number of Parabacteroides and Alistipes [28].

\section{Skin microbiota in psoriasis}

In general terms, four bacterial phyla dominate the healthy skin microbiota, namely Actinobacteria, Firmicutes, Proteobacteria, and Bacteroidetes, of which the genera Corynebacterium, Propionibacterium, and Staphylococcus are the most abundant [3, 9, 25]. In the skin microbiome, changes have been observed in the relative presence of Firmicutes, Actinobacteria, and Proteobacteria. Additionally, Staphylococcus and Streptococcus spp. were detected more frequently in skin lesions [6]. Firmicutes was found to be the most abundant phylum of bacteria in lesions from psoriatic skin, whereas Actinobacteria was significantly underrepresented in psoriatic skin lesions when compared to healthy and non-lesional skin [3].

Overall, the microbiome from PsO lesions is significantly different compared to healthy skin. In psoriatic lesions, Firmicutes was the most common phylum. Other studies have shown that PsO lesions were dominated by Proteobacteria and Bacteroidetes [6]. Additionally, Streptococcus spp. were detected more frequently in skin lesions [9]. This is linked to reports that Streptococcal $\mathrm{M}$ proteins may mimic keratin determinants and consequently this leads to T cell activation [41] in PsO.

\section{Parasites in psoriasis - better than a worse player}

Most infections with helminths are often asymptomatic [42]. But in extreme cases, long-term infections, depending on the host condition, may be responsible for clinical pathology such as the development of anemia, growth re- tardation, or even cognitive disturbances in children [43]. Indeed, soil-transmitted intestinal nematodes (Ascaris lumbricoides, Trichuris trichiura, Necator americanus) and intracellular parasites from the genus Acanthamoeba are still important health problems.

Parasitism affects the host organisms at many levels, and the parasite itself may also be a vector of bacteria or viruses, significantly affecting, both directly and indirectly, the host's microbiome. However, the frequency of the diseases induced by Acanthamoeba is low relative to the widespread distribution of Acanthamoeba; amoebas can influence the microbiota and affect many serious bacterial infections. Over one hundred microorganisms are transferred by amoebae, including Mycobacterium, Shigella, Salmonella, and Yersinia, and Bacillus anthracis, Vibrio cholerae, Legionella pneumophila, Francisella tularensis, and Coxiella burnetiid. As "Trojan horses" amoebae can influence the host macrobiome with other parasites: Cryptosporidium parvum, Toxoplasma gondii, and even the virus Pandoravirus inopinatum [44].

On the other hand, the proper development of immune mechanisms requires stimulation by particular components of the microbiome: bacteria, systemic commensal bacteria and viruses, parasites and fungi. Neonates and germ-free animals are characterized by an incompletely developed immune system, Th2-type responses predominate, and they exhibit reduced levels of B and T cells and also gut-associated immune responses. It means that parasitic antigens are important in immune system development and infection can influence the microbiome and in this way influence the immune response. In countries where there is a large number of parasitic worm infections, autoimmune and allergic diseases remain relatively rare $[11,45]$. For millennia human life coexisted with parasites, which developed mechanisms that allowed them to survive in the host [11]. Additionally, epidemiological studies have demonstrated an inverse correlation between rates of helminth infections and the prevalence of immune-mediated disease [42]. Economic development has increased the availability of anti-parasitic and anti-microbial drugs, which has directly reduced exposure to potential infections and is correlated with the increase in the incidence of autoimmune diseases in developed countries [45]. Therefore, it has been hypothesized that worms can protect against the development of autoimmune diseases and allergies [42, 46]. It is worth paying attention to the hygiene hypothesis and to the old friends hypothesis, which suggest that the absence or delay of certain infections, primarily in childhood, may result in an increase in the prevalence of chronic inflammatory disorders such as asthma, autoimmune diseases, and inflammatory bowel disease [2, 46]. The 'hygiene hypothesis' has been given an immunological framework in which the balance between type Th1 (associated with bacterial and viral infections and autoimmune diseases) and type Th2 (associated with helminth infections and allergic diseases) 
immune responses is pivotal. It has been postulated that limited exposure to bacterial and viral pathogens during early childhood results in insufficient stimulation of Th1 cells, which in turn cannot counterbalance the expansion of Th2 cells and results in a predisposition to allergy [11].

One of the most important features of nematodes is their longevity; the main factor enabling this is the suppression of the host immune system [11] through the production and secretion of different molecules, including proteins. Some helminth proteins can manipulate the host's immune system, a phenomenon that is now being exploited with a view to developing therapeutics for inflammatory diseases [4]. These studies suggest long evolutionary coadaptation between parasites and hosts, and a key to this partnership is the immunological interaction between them [4, 46].

Helminths have adapted to life in different environments, e.g. liver, intestine, lungs, blood vessels. For this purpose, helminths secrete a range of molecules that facilitate their penetration, migration, and establishment in the host [5]. Intestinal helminth are multi-cellular organisms that belong to distinct phyla: nematodes and flatworms [4]. One of the most important mechanisms enabling longterm host colonization is the immunomodulating effect of helminths. Immunological mechanisms provide a mutual benefit, protecting the host from the severe consequences of the inflammatory response and prevent the elimination of helminths. Helminths affect the human immune system by several mechanisms including induction of DC tolerogenic, Breg and Treg cells, which play a critical role in maintaining immune homeostasis. Increasing the number of regulatory cells lengthens parasite survival, and may protect against the development of or affect the course of certain autoimmune diseases. Many of these benefits are highlighted in several reviews and opinion pieces [47, 48]. Gastrointestinal helminth infection causes a bias towards Th2 and an increased Treg response. Tregs modulate both Th1 and Th2, and may have long-term effects because helminths can be transmitted to infants in utero [49]. Due to the importance of Th1 responses in controlling bacteria and protozoa infection, parasites seem to increase susceptibility to co-infection and reduce vaccine efficacy. In fact, parasite infection may partially explain the failure of BCG vaccination [50].

Interaction of the parasite and microbiome is important in analyzing helminth-induced immunomodulation. Parasites modify the microbiome and regulate the plasticity between Tregs and Th17 cells. Bacteria-induced Tregs express the receptor RORgT (Ror ${ }^{+}$Treg) and have the ability to differentiate into Th17 cells. The absence of these cells, during helminth infection, exacerbates the Th2 response. In contrast, Nippostrongylus brasiliensis infection contributes to reducing the abundance of SFB. These bacteria have been implicated in the induction of Th17 responses in inflammatory disorders $[2,51]$. These studies indicate how important is a balance in the microbiome, which al- lows appropriate immune responses at mucosal surfaces. The resulting dependency allows intestinal worms to detect commensal bacterial cues that inform them that they have reached an appropriate microenvironment for their development. Thus, they promote the expansion of bacteria that induce regulatory responses [2]. Studies have shown that Trichuris muris infection mainly modulated Bacteroidetes by reducing the diversity and abundance of Prevotella and Parabacteroides species. That perturbation of the microbiota was transitory and returned to normal upon clearance of the parasite. These studies showed, however, that chronic helminth infection can permanently affect immune system cell populations, which can have implications for future pathophysiology [1]. In general, not much research has been conducted on the crosstalk between intestinal parasites and skin disease. However, mice infected with the intestinal nematode Heligmosomoides polygyrus were less susceptible in a model of skin contact hypersensitivity. The mechanism is unknown but seems to be independent of Tregs [52].

In addition to the living worms, nematode products can play a central role in bidirectionally regulating the gut microbiome and training of immune responses, with a consequent impact on intestinal barrier integrity. Functional metagenomic analysis showed that the tissue-dwelling filarial nematode Acanthocheilonema viteae produces a phosphorylcholine (PC)-containing glycoprotein (ES-62) which perturbs the fatty acid, lipid and isoprenoid metabolic capacity of the microbiota in collagen-induced arthritis (CIA) mice [53]. Subcutaneous administration of ES-62 enriches butyrate-producing species to counter the outgrowth of pathogenic Escherichia and Helicobacter species and influences the development of lesions in the colon of CIA - a mouse model of rheumatoid arthritis - which is associated with normalization of gut microbiota and prevention of loss of intestinal barrier integrity. According to this hypothesis, nematodes can prevent or resolve chronic inflammation by sensing and normalizing the gut microbiome, promoting microbiome homeostasis and intestinal integrity [53].

Oral application of helminths raises ethical concerns that it can produce side-effects in long-term therapy and might be insufficient for patients suffering from some diseases. However, it seems justified based on experimental mouse models [48]. For example, there is no cure for PsO; it can only be controlled medically by using, e.g. corticosteroids, cytostatic drugs, UV therapies and topically applied vitamin D analogues [54].

\section{Summary}

Microbial antigens play a significant role in immune regulation. The composition of the gut microbiome can dramatically affect immune development. The altered immune reactivity could affect susceptibility to diseases, 
especially autoimmune disorders. In response to infections, the adaptive immune system recognizes specific antigens through cell surface receptors. Depending on whether they are opportunistic or pathogenic bacteria, naive T cells might differentiate into effector $\mathrm{T}$ cells or into regulatory $\mathrm{T}$ cells. Therefore, dysbiosis influences the local and systemic immune system. Although the question remains whether dysbiosis in autoimmune disease is a cause or a consequence of chronic inflammation, worm-induced immunoregulation could alleviate the course of PsO [42]. Understanding the mechanism by which the microbiome and macrobiome influence pathogenesis may prove crucial in the application of new therapies and innovative future solutions.

\section{Acknowledgements}

This work was supported by grants from the TEAM TECH/2017-4/22 project carried out within the TEAM TECH program of the Foundation for Polish Science co-financed by the European Union under the European Regional Development Fund.

The authors declare no conflict of interest.

\section{References}

1. Zhao Q, Elson CO (2018): Adaptive immune education by gut microbiota antigens. Immunology 154: 28-37.

2. Harnett MM, Harnett W (2017): Can parasitic worms cure the modern world's ills? Trends Parasitol 33: 694-705.

3. Yan D, Issa N, Afifi L, et al. (2017): The role of the skin and gut microbiome in psoriatic disease. Curr Dermatol Rep 6: 94-103.

4. Abu-Shakra M, Ling E, Shoenfeld Y (2015): Parasitic infection and autoimmunity. Infect Autoimmun, Elsevier Inc.; 621-641.

5. Khare S, Trivedi T (2020): Coexisting parasitic infestations in patients with psoriasis and effects of deworming therapy on response of treatment. Panacea J Med Sci 10: 68-70.

6. Benhadou F, Mintoff D, Schnebert B, et al. (2018): Psoriasis and microbiota: a systematic review. Diseases 6: 47.

7. Kim J, Krueger JG (2015): The immunopathogenesis of psoriasis. Dermatol Clin 33: 13-23.

8. World Health Organization (2016): Global report on. Glob Rep Psoriasis 978: 1-26.

9. Visser MJE, Kell DB, Pretorius E (2019): Bacterial dysbiosis and translocation in psoriasis vulgaris. Front Cell Infect Microbiol 9: 7.

10. Lowes MA, Kikuchi T, Fuentes-Duculan J, et al. (2008): Psoriasis vulgaris lesions contain discrete populations of Th1 and Th17 T cells. J Invest Dermatol 128: 1207-1211.

11. Wammes LJ, Mpairwe H, Elliott AM, et al. (2014): Helminth therapy or elimination: Epidemiological, immunological, and clinical considerations. Lancet Infect Dis 14: 1150-1162.

12. Boehncke WH (2015): Etiology and pathogenesis of psoriasis. Rheum Dis Clin North Am 41: 665-675.

13. Ogawa E, Sato Y, Minagawa A, et al. (2018): Pathogenesis of psoriasis and development of treatment. J Dermatol 45: 264-272.
14. Woo YR, Cho DH, Park HJ (2017): Molecular mechanisms and management of a cutaneous inflammatory disorder: psoriasis. Int J Mol Sci 18: 2684.

15. Bai F, Zheng W, Dong Y, et al. (2018): Serum levels of adipokines and cytokines in psoriasis patients: A systematic review and meta-analysis. Oncotarget 9: 1266-1278.

16. Rashmi R, Rao KSJ, Basavaraj KH (2009): A comprehensive review of biomarkers in psoriasis. Clin Exp Dermatol 34: 658-663.

17. Koo J, Marangell LB, Nakamura M, et al. (2017): Depression and suicidality in psoriasis: review of the literature including the cytokine theory of depression. J Eur Acad Dermatology Venereol 31: 1999-2009.

18. Sweeney CM, Tobin AM, Kirby B (2011): Innate immunity in the pathogenesis of psoriasis. Arch Dermatol Res 303: 691-705.

19. Büchau AS, Gallo RL (2007): Innate immunity and antimicrobial defense systems in psoriasis. Clin Dermatol 25: 616-624.

20. Bassukas I, Gaitanis G, Katsanos K, et al. (2016): Psoriasis and inflammatory bowel disease: links and risks. Psoriasis Targets Ther Volume 6: 73-92.

21. Kim J, Krueger JG (2017): Highly effective new treatments for psoriasis target the IL-23/type $17 \mathrm{~T}$ cell autoimmune axis. Annu Rev Med 68: 255-269.

22. Koboziev I, Reinoso Webb C, Furr KL, et al. (2014): Role of the enteric microbiota in intestinal homeostasis and inflammation. Free Radic Biol Med 68: 122-133.

23. Viggiano D, Ianiro G, Vanella G, et al. (2015): Gut barrier in health and disease: Focus on childhood. Eur Rev Med Pharmacol Sci 19: 1077-1085.

24. Lopetuso LR, Scaldaferri F, Bruno G, et al. (2015): The therapeutic management of gut barrier leaking: The emerging role for mucosal barrier protectors. Eur Rev Med Pharmacol Sci 19: 1068-1076.

25. Wang WM, Jin HZ (2018): Skin microbiome: an actor in the pathogenesis of psoriasis. Chin Med J (Engl) 131: 95-98.

26. Ren J, Li B (2017): The functional stability of FOXP3 and ROR $\gamma \mathrm{t}$ in Treg and Th17 and their therapeutic applications. Adv Protein Chem Struct Biol 107: 155-189.

27. Shapiro H, Thaiss CA, Levy M, et al. (2014): The cross talk between microbiota and the immune system: Metabolites take center stage. Curr Opin Immunol 30: 54-62.

28. Hidalgo-Cantabrana C, Gómez J, Delgado S, et al. (2019): Gut microbiota dysbiosis in a cohort of patients with psoriasis. Br J Dermatol 181: 1287-1295.

29. Doré J, Corthier G (2010): Le microbiote intestinal humain. Gastroenterol Clin Biol 34: S7-S15.

30. Chang HW, Yan D, Singh R, et al. (2018): Alteration of the cutaneous microbiome in psoriasis and potential role in Th17 polarization. Microbiome 6: 1-27.

31. Kabat AM, Srinivasan N, Maloy KJ (2014): Modulation of immune development and function by intestinal microbiota. Trends Immunol 35: 507-517.

32. Benhadou F, Mintoff D, Del Marmol V (2018): Psoriasis: keratinocytes or immune cells - which is the trigger? Dermatology 1-10.

33. Scher JU, Ubeda C, Artacho A, et al. (2015): Decreased bacterial diversity characterizes an altered gut microbiota in psoriatic arthritis and resembles dysbiosis of inflammatory bowel disease. Arthritis Rheumatol 67: 128-139.

34. Shapiro J, Cohen NA, Shalev V, et al. (2019): Psoriatic patients have a distinct structural and functional fecal microbiota compared with controls. J Dermatol 46: 595-603.

35. Codońer FM, Ramírez-Bosca A, Climent E, et al. (2018): Gut microbial composition in patients with psoriasis. Sci Rep 8: 1-7. 
36. Doaa M, Dalia M, Ahmed FS (2016): Gut bacterial microbiota in psoriasis: A case control study. African J Microbiol Res 10: $1337-1343$.

37. Ferreira CM, Vieira AT, Vinolo MAR, et al. (2014): The central role of the gut microbiota in chronic inflammatory diseases. J Immunol Res 2014: 689492.

38. Zheng H, Liang H, Wang Y, et al. (2016): Altered gut microbiota composition associated with eczema in infants. PLoS One 11: 1-13.

39. Vinolo MAR, Rodrigues HG, Nachbar RT, et al. (2011): Regulation of inflammation by short chain fatty acids. Nutrients 3: 858-876.

40. Eppinga H, Fuhler GM, Peppelenbosch MP, et al. (2016): Gut microbiota developments with emphasis on inflammatory bowel disease: report from the gut microbiota for health world summit 2016. Gastroenterology 151: e1-e4.

41. Owczarczyk-Saczonek A, Placek W (2014): Psoriasis as an autoimmune disease. Przegl Dermatol 101: 278-287.

42. Greb J, Gottlieb A (2013): Review worms: A remarkably reasonable treatment option for psoriasis corresponding author disclosures. Psoriasis Forum 19: 94-97.

43. Ontaneda D, Cohen JA (2020): Keep the worms in the mud. JAMA Neurol 77: 1066-1067.

44. Borecka A, Bielawska-Drózd A, Skotarczak B, et al. (2020): Acanthamoeba - pathogen and vector of highly pathogenic bacteria strains to healthy and immunocompromised individuals. Cent Eur J Immunol 45: 228-232.

45. Yazdanbakhsh M, Kremsner PG, Van Ree (2002): Allergy, parasites, and the hygiene hypothesis. Science 296: 490-494.

46. Sotillo J, Toledo R, Mulvenna J, et al. (2017): Exploiting helminth-host interactomes through big data. Trends Parasitol 33: 875-888.

47. Maruszewska-Cheruiyot M, Donskow-Łysoniewska K, Doligalska M (2019): Helminth therapy - local and systemic activity, on example of inflammatory bowel diseases and multiple sclerosis. Postepy Hig Med Dosw 73: 645-653.

48. Maruszewska-Cheruiyot M, Donskow-Lysoniewska K, Doligalska M (2018): Helminth therapy: Advances in the use of parasitic worms against inflammatory bowel diseases and its challenges. Helminthol 55: 1-11.

49. Blackwell AD (2016): Helminth infection during pregnancy: Insights from evolutionary ecology. Int J Womens Health 8: 651-661.

50. Wait LF, Dobson AP, Graham AL (2020): Do parasite infections interfere with immunisation? A review and meta-analysis. Vaccine 38: 5582-5590.

51. Houlden A, Hayes KS, Bancroft AJ, et al. (2015): Chronic Trichuris muris infection in C57BL/6 mice causes significant changes in host microbiota and metabolome: Effects reversed by pathogen clearance. PLoS One 10: e.0125945.

52. Filbey KJ (2020): The gastrointestinal helminth heligmosomoides bakeri suppresses inflammation in a model of contact hypersensitivity. Front Immunol 11: 950.

53. Doonan J, Tarafdar A, Pineda MA, et al. (2019): The parasitic worm product ES-62 normalises the gut microbiota bone marrow axis in inflammatory arthritis. Nat Commun 10: 1554.

54. Deng Y, Chang C, Lu Q (2016): The inflammatory response in psoriasis: a comprehensive review. Clin Rev Allergy Immunol 50: $377-389$. 